\title{
Erratum to: \\ Progressive Supranuclear Palsy
}

\author{
Maria Stamelou and Wolfgang $\mathrm{H}$. Oertel
}

\author{
(C) Springer-Verlag Wien 2017 \\ C. Falup-Pecurariu et al. (eds.), Movement Disorders Curricula, \\ DOI 10.1007/978-3-7091-1628-9_18
}

The author missed to add the source line to the Fig. 18.1 and the correct figure legend should read as:

Fig. 18.1 Tau pathology in a postmortem brain of a PSP patient within the basal ganglia. Immunostaining with the AD2 antibody against human tau. Pathological AD2 immunoreaction in cytoplasm of oligodendrocytes (coiled body) and astrocytes (tuff), in neurites (threads), and in neuronal cytoplasm in loose (pre-tangle) and dense aggregation (tangle) (Stamelou et al, 2010) (Photos: G. Hoeglinger)

The updated online version of the original chapter can be found under DOI 10.1007/978-3-7091-1628-9_18

\footnotetext{
M. Stamelou, MD, PhD $(\bowtie)$

Department of Neurology, University Clinic,

Philipps University Marburg,

Baldingerstrasse 35039, Germany
}

Movement Disorders Department, Hygeia Hospital,

Erythrou Stavrou 4 \& Kifisiaw, Marousi, Athens

15123, Greece

Second Department of Neurology,

Kapodistrian University of Athens, Rimini 1,

Athens 12462, Greece

e-mail: mariastamelou@gmail.com

W.H. Oertel, MD

Department of Neurology, University Clinic,

Philipps University Marburg,

Baldingerstrasse 35039, Germany

e-mail: oertelw@med.uni-marburg.de 